\title{
Hamilton-Jacobi and Schrodinger Separable Solutions of Einstein's Equations
}

\author{
BRANDON CARTER \\ Department of Applied Mathematics and Theoretical Physics \\ Cambridge, England
}

Received April 17, 1968

\begin{abstract}
This paper contains an investigation of spaces with a two parameter Abelian isometry group in which the Hamilton-Jacobi equation for the geodesics is soluble by separation of variables in such a way that a certain natural canonical orthonormal tetrad is determined. The spaces satisfying the stronger condition that the corresponding Schrodinger equation is separable are isolated in a canonical form for which Einstein's vacuum equations and the source-free Einstein-Maxwell equations (with or without a $\Lambda$ term) can be solved explicitly. A fairly extensive family of new solutions is obtained including the previously known solutions of de Sitter, Kasner, Taub-NUT, and Kerr as special cases.
\end{abstract}

\section{Introduction}

Robertson [1] and Eisenhart [2] have discussed conditions under which the Hamilton-Jacobi equation and the corresponding Schrodinger equation are soluble by separation of variables in spaces which admit a complete set of mutually orthogonal families of hypersurfaces. This paper also contains a study of spaces with separable Hamilton-Jacobi and Schrodinger equations but under different conditions: firstly, the investigation is restricted to ordinary four-dimensional space-time, while being extended to include the case where the Hamilton-Jacobi and Schrodinger equations under consideration apply not only to the motions of free particles (geodesics) but also to the motions of charged particles in a Maxwell field; secondly there is the more important difference that instead of requiring that the families of hypersurfaces with respect to which separation takes place be all mutually orthogonal, we require that there be an Abelian isometry group, and that the separation should take place in such a way as to define a certain canonical tetrad of orthonormal (and not necessarily integrable) forms.

One of the purposes of requiring these separability properties for a space is to obtain sufficiently strong restrictions on it to make detailed study possible, but without imposing a high isometry group. Most of the spaces which have been studied in detail in the past have had at least three parameter isometry groups. The type of separability to be discussed here applies to spaces with (locally) two parameter Abelian iso- 
metry groups. Another reason for imposing these requirements in that many of the most interesting global properties of spaces depend on the behaviour of geodesics, and cannot be studied easily unless at least first integrals of the geodesic equations can be found. If the space has an isometry group of three or more parameters then a complete set of four such integrals can always be found, namely the three corresponding generalised momenta together with the derivative of metric distance with respect to affine distance (which is constant of the motion in any case, independently of any isometry). However if the isometry group has no more than two parameters, a complete set of first integrals cannot be found except in special circumstances, of which the only widely useful case is that where the Hamilton-Jacobi equation is separable.

We shall be led to impose the considerably stronger condition that the analogous Schrodinger equation is separable not because there is any good physical reason for doing so but because it leads to a very simple algebraical form for the metric, namely

$$
d s^{2}=\frac{Z}{\Delta_{\lambda}} d \lambda^{2}+\frac{Z}{\Delta_{\mu}} d \mu^{2}+\frac{\Delta_{\mu}}{Z}\left[P_{\lambda} d \psi-Q_{\lambda} d \chi\right]^{2}-\frac{\Delta_{\lambda}}{Z}\left[P_{\mu} d \psi-Q_{\mu} d \chi\right]^{2}
$$

where $\psi, \chi$ are two ignorable co-ordinaters and $\lambda, \mu$ are two nonignorable co-ordinates and where $\Delta_{\lambda}, P_{\lambda}, Q_{\lambda}$ are functions of $\lambda$ only and $\Delta_{\mu}, P_{\mu}, Q_{\mu}$ are functions of $\mu$ only. The quantity $Z$ is defined to be the determinant

$$
Z=P_{\lambda} Q_{\mu}-P_{\mu} Q_{\lambda}
$$

and it is necessary that the functions $P_{\lambda}, P_{\mu}, Q_{\lambda}, Q_{\mu}$ be such that $Z$ takes the form of a sum of quantities each depending on only one of the variables $\lambda, \mu$. Apart from this restriction (which means that only two of them can be chosen independently) these four functions are arbitrary, as are the two functions $\Delta_{\lambda}, \Delta_{\mu}$. The simplest Maxwell field which preserves the separability may be derived from a covariant vector potential which in these co-ordinates takes the form

$$
A=\frac{P_{\lambda} X_{\mu}+P_{\mu} X_{\lambda}}{Z} d \psi-\frac{Q_{\lambda} X_{\mu}+Q_{\mu} X_{\lambda}}{Z} d \chi,
$$

where $X_{\lambda}, X_{\mu}$ are two further arbitrary functions of the single variables $\lambda, \mu$ respectively.

The final task of this paper is to obtain the complete solution of Einstein's vacuum equations (both with and without $\Lambda$ term) and of the Einstein-Maxwell equations for the above system. The solutions are obtained in a simple and explicit form, and fall into three families: firstly the most general family $[A]$, where $Z$ depends on both $\lambda$ and $\mu$; secondly a more restricted family $[\widetilde{B}]$, where $Z$ depends on only one of these variables, and which divides into two conjugate subfamilies 
$\left[\widetilde{B}_{-}(+)\right]$where the dependence is on $\lambda$ only, and $[\widetilde{B}(-)]$ where the dependence is on $\mu$ only; and thirdly a degenerate family $[D]$ for which $Z$ is constant. These families may be tabulated as follows:

$$
\begin{aligned}
d s^{2}=\left(\lambda^{2}\right. & \left.+\mu^{2}\right)\left\{\frac{d \lambda^{2}}{\Delta_{\lambda}}+\frac{d \mu^{2}}{\Delta_{\mu}}\right\} \\
& +\frac{\Delta_{\mu}\left[d \chi-\lambda^{2} d \psi\right]^{2}-\Delta_{\lambda}\left[d \chi+\mu^{2} d \psi\right]^{2}}{\lambda^{2}+\mu^{2}},
\end{aligned}
$$

where

$$
\begin{aligned}
& \Delta_{\lambda}=\frac{1}{3} \Lambda \lambda^{4}+h \lambda^{2}-2 m \lambda+p+e^{2}, \\
& \Delta_{\mu}=\frac{1}{3} \Lambda \mu^{4}-h \mu^{2}+2 q \mu+p,
\end{aligned}
$$

and where

$$
\begin{aligned}
A & =e\left\{\frac{\lambda \mu(\mu \cos \alpha+\lambda \sin \alpha)}{\lambda^{2}+\mu^{2}} d \psi+\frac{\lambda \cos \alpha-\mu \sin \alpha}{\lambda^{2}+\mu^{2}} d \chi\right\} . \\
{[\widetilde{B}(+)] \quad d s^{2} } & =\left(\lambda^{2}+l^{2}\right)\left\{\frac{d \lambda^{2}}{\Delta_{\lambda}}+\frac{d \mu^{2}}{\Delta_{\mu}}+\Delta_{\mu} d \psi^{2}\right\}-\frac{\Delta_{\lambda}[d \chi+2 l \mu d \psi]^{2}}{\lambda^{2}+l^{2}}
\end{aligned}
$$

where

$$
\begin{gathered}
\Delta_{\lambda}=\Lambda\left(\frac{1}{3} \lambda^{4}+2 l^{2} \lambda^{2}-l^{4}\right)+h\left(\lambda^{2}-l^{2}\right)-2 m \lambda+e^{2} \\
\Delta_{\mu}=-h \mu^{2}+2 q \mu+p
\end{gathered}
$$

and where

$$
\begin{aligned}
A & =e\left\{\frac{\mu\left(2 l \lambda \cos \alpha+\left(\lambda^{2}+l^{2}\right) \sin \alpha\right]}{\lambda^{2}+l^{2}} d \psi+\frac{\lambda \cos \alpha}{\lambda^{2}+l^{2}} d \chi\right\} . \\
{[\widetilde{B}(-)] \quad d s^{2} } & =\left(\mu^{2}+k^{2}\right)\left\{\frac{d \lambda^{2}}{\Delta_{\lambda}}+\frac{d \mu^{2}}{\Delta_{\mu}}-\Delta_{\lambda} d \psi^{2}\right\}+\frac{\Delta_{\mu}[d \chi-2 k \lambda d \psi]^{2}}{\mu^{2}+k^{2}}
\end{aligned}
$$

where

$$
\begin{aligned}
& \Delta_{\lambda}=h \lambda^{2}-2 m \lambda+n \\
& \Delta_{\mu}=\Lambda\left(\frac{1}{3} \mu^{4}+2 k^{2} \mu^{2}-k^{4}\right)-h\left(\mu^{2}-k^{2}\right)+2 q \mu-e^{2}
\end{aligned}
$$

and where

$$
\begin{gathered}
A=e\left\{\frac{\lambda\left[\left(\mu^{2}+k^{2}\right) \cos \alpha+2 k \mu \sin \alpha\right]}{\mu^{2}+k^{2}} d \psi+\frac{\mu \sin \alpha}{\mu^{2}+k^{2}} d \chi\right\} . \\
d s^{2}=\frac{d \lambda^{2}}{\Delta_{\lambda}}+\frac{d \mu^{2}}{\Delta_{\mu}}+\Delta_{\mu} \alpha \chi^{2}-\Delta_{\lambda} d \psi^{2}
\end{gathered}
$$

where

and where

$$
\begin{aligned}
& \Delta_{\lambda}=\left(\Lambda+e^{2}\right) \lambda^{2}-2 m \lambda+n \\
& \Delta_{\mu}=\left(\Lambda-e^{2}\right) \mu^{2}+2 q \mu+p
\end{aligned}
$$

$$
A=e\{\lambda \cos \alpha d \psi-\mu \sin \alpha d \chi\} .
$$

In all of these cases the charge parameter $e$ and the other parameters $h$, $k, l, m, n, q, p$ determining the metric forms are arbitrary except that they and the co-ordinate $\mu$ must lie within the range where the quantity 
$\Delta_{\mu}$, (which determines the over-all signature of the metric) is positive. The parameter $\alpha$ is an arbitrary angle determining the complexion of the electromagnetic field, and it has no effect on the metric.

This search was prompted by the author's observation [3] that in the Kerr solution and its charged generalisation the Hamilton-Jacobi equation is separable in the simple manner postulated here, and since it turns out that the Schrodinger equation is also separable in these solutions, they must be included in the above family. In fact they constitute the special case of the set $[A]$ for which $\Lambda$ and $q$ vanish and for which $h$ and $n$ are both (strictly) positive. The other special cases where the metrics given above coincide with previously known solutions are enumerated in the final section of this paper, but more general investigation of the properties of these solutions is left for a subsequent discussion.

The forms $[\widetilde{B}( \pm)]$, although superficially more complicated then $[A]$, are actually merely limiting special cases of this more general form; moreover the form $[D]$ is obtainable by a further limiting process from either $[\widetilde{B}(+)]$ or $[\widetilde{B}(-)]$. This is explicitly demonstrated in the final section where the form $[A]$ is analytically extended to a form $[\widetilde{A}]$ which combines $[A],[\widetilde{B}(+)],[\widetilde{B}(-)]$ and $[D]$ in a single formula. The inclusive solution $[\widetilde{A}]$ is the form given by the author [4] without derivation in a previous note.

\section{The Hamilton-Jacobi and Schrodinger Equations}

Before describing the separability conditions which we shall use, we must specify the precise form of the Hamilton-Jacobi and Schrodinger equations to which we refer.

We start by considering a general space with co-ordinates $x^{\alpha}$ and metric (with positive Lorentz signature)

$$
d s^{2}=g_{\alpha \beta} d x^{\alpha} d x^{\beta},
$$

on which there is an electromagnetic field

$$
F=F_{\alpha \beta} d x^{\alpha} \wedge d x^{\beta},
$$

satisfying the Maxwell equation

$$
d F=0 .
$$

The equation of motion of a particle of mass $\bar{m}$ and charge $\bar{e}$ is

$$
g_{\alpha \beta} \frac{d^{2} x^{\beta}}{d s^{2}}-\left[\frac{1}{2} \frac{\partial g_{\beta \gamma}}{\partial x^{\alpha}}-\frac{\partial g_{\alpha \beta}}{\partial x^{\gamma}}\right] \frac{d x^{\beta}}{d s} \frac{d x^{\gamma}}{d s}=\frac{\bar{e}}{\bar{m}} F_{\alpha \beta} \frac{d x^{\beta}}{d s}
$$

which reduces to the geodesic equation when $\bar{e}$ vanishes.

In consequence of Maxwell's equation (22) we can introduce a potential

$$
A=A_{\alpha} d x^{\alpha}
$$


satisfying

$$
F=2 d A
$$

and in terms of this we can construct the Lagrangian

$$
L=\frac{1}{2} g_{\alpha \beta} \dot{x}^{\alpha} \dot{x}^{\beta}+\bar{e} A_{\alpha} \dot{x}^{\alpha}
$$

where - denotes ordinary differentiation with respect to an affine parameter $\tau$. This Lagrangian gives rise to the equation of motion (23) provided that $\tau$ is normalised with respect to the proper distance $s$ by

$$
s=\bar{m} \tau,
$$

which is equivalent to imposing the normalising condition

$$
g_{\alpha \beta} \dot{x}^{\alpha} \dot{x}^{\beta}=-\bar{m}^{2} \text {. }
$$

When there is no charge the normalisation is unimportant except in sign: positive, zero, and negative values of $\bar{m}^{2}$ give respectively timelike, null, and spacelike geodesics.

Introducing the momenta

$$
P_{\alpha}=g_{\alpha \beta} \dot{x}^{\beta}+\bar{e} A_{\alpha}
$$

derived from (26), and the inverse metric tensor

$$
\left(\frac{\partial}{\partial s}\right)^{2}=g^{\alpha \beta}\left(\frac{\partial}{\partial x^{\alpha}}\right)\left(\frac{\partial}{\partial x^{\beta}}\right)
$$

we obtain the Hamiltonian

$$
H=\frac{1}{2} g^{\alpha \beta}\left(p_{\alpha}-\bar{e} A_{\alpha}\right)\left(p_{\beta}-\bar{e} A_{\beta}\right) .
$$

Since it does not involve the affine parameter explicitly, $H$ is itself a constant of the motion, and in consequence of (28) its value is given by

$$
H=-\frac{1}{2} \bar{m}^{2} \text {. }
$$

Eq. (31) gives rise immediately to the Hamilton-Jacobi equation

$$
\frac{\partial S}{\partial \tau}=\frac{1}{2} g^{\alpha \beta}\left(\frac{\partial S}{\partial x^{\alpha}}-\bar{e} A_{\alpha}\right)\left(\frac{\partial S}{\partial x^{\beta}}-\bar{e} A_{\beta}\right)
$$

where $S$ is the Jacobi action, from which, once it is known, the integrated form of the corresponding geodesics or orbits can be obtained automatically by the standard methods.

We may proved to the quantised analogue of the theory in the normal way by treating the Hamiltonian and the momenta as operators. Thus (31) becomes a Schrodinger equation if we make the substitutions

$$
H=i \frac{\partial}{\partial \tau} ; \quad p_{\alpha}=i \frac{\partial}{\partial x^{\alpha}},
$$


and allow it to act on a scalar wave function $\Psi$. Since the operators do not commute the order must be determined by invariance requirements. It may easily be verified that the equation

$$
i \frac{\partial \Psi}{\partial \tau}=\left(|g|^{-1 / 2} \frac{i \partial}{\partial x^{\alpha}}|g|^{1 / 2}-\bar{e} A_{\alpha}\right) \frac{1}{2} g^{\alpha \beta}\left(\frac{i \partial}{\partial x^{\beta}}-\bar{e} A_{\beta}\right) \Psi
$$

with the abbreviation

$$
g=\operatorname{det}\left(g_{\alpha \beta}\right)
$$

is in fact invariant under co-ordinate transformations and under the gauge transformations

$$
A \rightarrow A+\bar{e}^{-1} d \chi ; \quad \Psi \rightarrow \Psi e^{-i \chi},
$$

where $\chi$ is any real scalar field independent of $\tau$. Thus (35) is the Schrodinger equation analogous to the Hamilton-Jacobi equation (33). This formalism represents a quantisation over the five dimensional space consisting of the product of the original space-time manifold with the proper time (or affine distance) line. The eigenstates of the Hamiltonian take the form

$$
\Psi=e^{\frac{1}{2} i \bar{m} s} \psi,
$$

where $\psi$ is independent of the proper time $s$ and satisfies the equation

$$
\left(|g|^{-1 / 2} \frac{i \partial}{\partial x^{\alpha}}|g|^{1 / 2}-\bar{e} A_{\alpha}\right) g^{\alpha \beta}\left(\frac{i \partial}{\partial x^{\beta}}-\bar{e} A_{\beta}\right) \psi+\bar{m}^{2} \psi=0,
$$

which is simply the generalisation to curved space and charged particles of the Klein-Gordon equation. (It is worth remarking that by this method of derivation the generalisation is obtained uniquely, i.e. it would not be permissible for example to introduce the additional term $1 / 6 R \psi$ where $R$ is the curvature scalar, which would be required in order to make the equation conformally invariant.) Five dimensional proper time quantisation schemes of this sort in flat space have been discussed by NamBU [5], Feymmann [6] and GaRRod [7], but for the purposes of this investigation the physical interpretation is not important.

\section{The Separability Conditions}

In this section we shall describe the isometry and separability conditions which will be imposed, and examine their immediate consequences.

The first condition to be imposed is:

I. The space and the electromagnetic field are invariant under a two parameter Abelian symmetry group.

This means that we can introduce a co-ordinate system with coordinates $\lambda^{i}, \varphi^{2 r}$, in which the labels $i, r$ run over the values \pm 1 in such a way that the co-ordinates $\lambda^{i}$ are constant on the surfaces of transitivity, while the co-ordinates $\varphi^{2 r}$ are dragged along by the operations 20 Commun.math. Phys., Vol.10 
of the group, and so are ignorable in the sense that they do not appear in the expressions for the metric tensor or the Lagrangian or the Hamiltonian.

It is convenient to let the indices run over the values $\pm 1, \pm 2$, instead of running over consecutive integers as would be conventional, because this makes possible a concise notation in which the index variables may be used as multiplying factors to control signs. When used in this way an index will always be bracketed and it will be understood that it does not take part in the summation convention (e.g. (i) $\lambda^{i}$ means $\lambda^{1}$ or $-\lambda^{-1}$ in the respective cases $i=1, i=-1$ ). Thus the symbol $i$ bracketed in this way need not be confused with the unbracketed symbol $i$ used to denote the square root of minus one.

The summation convention will in any case be suspended for the labels of the non-ignorable co-ordinates (for which the symbols $i, j$ will be reserved) throughout this paper i.e. it is to be understood that there will be no summations over them except those stated explicitly. For all other indices and in particular for the labels of the ignorable co-ordinates (for which the symbols $r, s$ will be reserved) and for labels running over all four co-ordinates values $\pm 1, \pm 2$ (for which Greek symbols will always be used) the use of the summation convention is to be taken for granted.

The second condition to be imposed is:

II. The symmetry group is invertible with non null surfaces of transitivity.

By invertibility of the group (c. f. CARTER [8]) it is meant that in addition to the continuous symmetry group there is a discrete symmetry under simultaneous reversal of the directions of the Killing vector fields generating the continuous isometries. It is shown in the above reference that the invertibility implies that the group is orthogonally transitive, i.e. that the 2 -surfaces of transitivity of the group are orthogonal to a second family of 2 -surfaces. This means that it is possible to choose the ignorable co-ordinates $\varphi^{2 r}$ to be constant on these orthogonal 2-surfaces. The assumption that the surfaces of transitivity are non-null guarantees that we can obtain a locally non-degenerate co-ordinate system in this way. In such a co-ordinate system the invertibility is manifest in the absence of cross components of the metric tensor between the ignorable and non ignorable co-ordinates.

Thus in the co-ordinate system so defined the Hamilton-Jacobi equation (33) splits up in the form

$$
\begin{aligned}
2 \frac{\partial S}{\partial \tau}= & \sum_{i, j} g^{i j}\left(\frac{\partial S}{\partial \lambda^{i}}-\bar{e} A_{i}\right)\left(\frac{\partial S}{\partial \lambda^{j}}-\bar{e} A_{j}\right) \\
& +g^{2 r, 2 s}\left(\frac{\partial S}{\partial \varphi^{2 r}}-\bar{e} A_{2 r}\right)\left(\frac{\partial S}{\partial \varphi^{2 s}}-\bar{e} A_{2 s}\right) .
\end{aligned}
$$


We shall be concerned ultimately with solutions of the vacuum Einstein and Einstein-Maxwell equations, and for such spaces the results of Papapetrou [9] and CARTER [8] show that the space must automatically be invertible wherever the surfaces of transitivity are non-null in any region connected to a locus where one of the killing vectors generating the group vanish, as on a symmetry axis. Thus if the isometry group postulated in condition (I) corresponds to the space-time being stationary and axisymmetric (these being the most physically natural circumstances which would produce a two parameter Abelian group), then condition (II) imposes no further restriction at all as far as solution of the vacuum Einstein and Einstein-Maxwell equations are concerned.

We now introduce the primary separability condition:

III. The Hamilton-Jacobi equation is soluble by separation of variables in the simplest possible way, i.e. the solution $S$ takes the form of a sum of terms depending on one variable only, and on substitution of this form, and if necessary after multiplication of the whole equation by a suitable separating factor, the Hamilton-Jacobi equation breaks up into a sum of terms each depending on only one of the non-ignorable co-ordinates.

The dependence of $S$ on the affine distance and on the ignorable co-ordinates can be obtained at once. Choosing the $\lambda^{i}$ to be the nonignorable co-ordinates in terms of which separation takes place we obtain the form

$$
S=-\frac{1}{2} \bar{m}^{2} \tau+\Phi_{2 r} \varphi^{2 r}+\sum_{i} S_{i}\left(\lambda^{i}\right)
$$

where the constants $\Phi_{2 r}$ are the values of the conserved momenta $p_{2 r}$ and where each function $S$ depends only on the single variable $\lambda^{i}$. From now on any function to which a single suffix $i$ is attached will by implication be a function of $\lambda^{i}$ only, and a prime will be used to denote total differentiation of such a function with respect to $\lambda^{i}$.

On substituting (41) into (40) we obtain

$$
\begin{aligned}
-\bar{m}^{2} U= & \sum_{i, j} U g^{i j}\left(S_{i}^{\prime}-\bar{e} A_{i}\right)\left(S_{j}^{\prime}-\bar{e} A_{j}\right) \\
& +U g^{2 r, 2 s}\left(\Phi_{2 r}-\bar{e} A_{2 r}\right)\left(\Phi_{2 s}-\bar{e} A_{2 s}\right),
\end{aligned}
$$

where we have introduced an as yet arbitrary multiplying function $U$.

Condition III states that it is possible to choose $U$ so that this equation breaks up into a sum of terms depending on one or other of the variables $\lambda^{i}$ only (independently of the values of $\Phi_{2 r}$ ). It is clear that the left hand side will have this property only if $U$ itself does, i.e. we must have

$$
U=\sum_{i} U_{i}\left(\lambda^{i}\right)
$$

It should be noted that in an equation of this type, of which we shall have several, the function on the left hand side determines the functions $20^{*}$ 
on the right hand side only up to an additive constant which may be transferred between them. We can also deduce at once that we must have

$$
g^{i j}=0, \quad(i \neq j),
$$

since otherwise the presence of a term containing the cross product $S_{i}^{\prime} S_{j}^{\prime}$ would be inevitable. A mixed term involving $S_{i}^{\prime 2}$ can only be avoided if the coefficient of $S_{i}^{\prime 2}$ is a function of $\lambda^{i}$ only, i.e. we must have

$$
U g^{i i}=\Delta_{i}\left(\lambda^{i}\right) \text {. }
$$

[It may be remarked at this point that by a co-ordinate transformation in which $\lambda^{i}$ is replaced by a function of itself, $\Delta_{i}$ would be altered by a factor which could be any strictly positive function of $\lambda^{i}$, and we might be tempted to make such a transformation in order to set $\left|\Delta_{i}\right|$ equal to unity. However we shall retain the functions $\Delta_{i}$ as freely adjustable quantities, since it will turn out later that a slightly less obvious normalisation is much more convenient.]

When $\bar{e}$ vanishes, the only remaining terms in (42) which have not yet been considered are those involving $\Phi_{2 r} \Phi_{2 s}$ and in order that they shall break up in the required manner we need

$$
U g^{2 r 2 s}=W^{r s},
$$

where the functions $W^{r s}$ take the form

$$
W^{r s}=\sum_{i} W_{i}^{r s}\left(\lambda^{i}\right) .
$$

The conditions (43) to (47) are necessary and sufficient for a metric satisfying (I) and (II) also to satisfy (III) when the charge $\bar{e}$ vanishes. The metric is fully determined by the ten single variable functions $U_{i}$, $\Delta_{i}, W_{i}^{r s}$, all of which are unrestricted in form, the covariant metric tensor being given by

$$
d s^{2}=U\left\{\sum_{i} \frac{\left(d \lambda^{i}\right)^{2}}{\Delta_{i}}+W_{r s} d \psi^{2 r} d \varphi^{2 s}\right\},
$$

where we have introduced the notation $W_{r s}$ for the inverse matrix of $W^{r s}$.

In order that the separability should continue to hold when the charge $\bar{e}$ is present, we need restrictions on the vector potential. The term linear in $S_{i}^{\prime}$, namely $-2 \bar{e} \Delta_{i} A_{i} S_{i}^{\prime}$ can be a sum of unmixed terms only if $A_{i}$ is a function of $\lambda^{i}$ only. If $A_{i}$ is a function of $\lambda^{i}$ only, it gives no contribution to $F$, and therefore without loss of generality, by a gauge transformation, we may express this condition in the form

$$
A_{i}=0 \text {. }
$$

The terms involving the momenta $\Phi_{2 r}$ will be unmixed for all values of these momenta only if

$$
A_{2 r}=W_{r s} Y^{s},
$$


where the functions $Y^{r}$ have the form

$$
Y^{r}=\sum_{i} Y_{i}^{r}\left(\lambda^{i}\right) \text {. }
$$

The only term which remains to be considered is the one quadratic in the potential, which will have the required form only if

where $\Omega$ has the form

$$
W_{r s} Y^{r} Y^{s}=\Omega \text {, }
$$

$$
\Omega=\sum_{i} \Omega_{i}\left(\lambda^{i}\right)
$$

Thus (49) to (51) are necessary and sufficient conditions for a Maxwell field in the space with metric (48) to continue to satisfy the separability condition III in the presence of charge. The field is explicitly determined by (49) and (50) in terms of the four single variable functions $Y_{i}^{r}$, which however are not arbitrary but must satisfy the stringent conditions (52) and (53).

For general values of the functions $W_{i}^{r s}$ the system (51) to (53) is so restrictive that the only possible solution is the trivial one where the functions $Y_{i}^{r}$ vanish, i.e. where the Maxwell field vanishes. However, in special cases wider classes of solution will be possible. Condition (IV) will in fact give one of the simplest restrictions on the quantities $W^{r s}$ (short of making them all functions of one variable only) which makes nontrivial solutions possible.

$I V$. The separation required by condition III takes place in such a way that the terms containing derivatives with respect to the ignorable coordinates separate as the sum of two squares each depending on only one of the non-ignorable co-ordinates.

It is clear from Eqs. (40), (42), (46), (47) that in so far as the case where the charge vanishes is concerned, condition IV is equivalent to the pair of equations

$$
W_{i}=0,
$$

where we have made the definitions

$$
W_{i}=\operatorname{det}\left(W_{i}^{r s}\right),
$$

since the Eq. (35) are necessary and sufficient for it to be possible to write $W_{i}^{r s}$ in the form

$$
W_{i}^{r s}=\varepsilon_{i} V^{r}{ }_{i}\left(\lambda^{i}\right) V^{s}{ }_{i}\left(\lambda^{i}\right),
$$

where $\varepsilon_{i}$ is an indicator taking one of the values \pm 1 according to the sign of the trace $W_{i}^{r r}$ in order that the new single variable functions $V_{i}^{r}$ shall be real. On substitution of (56), the contravariant metric tensor takes the extremely simple form

$$
\left(\frac{\partial}{\partial s}\right)^{2}=\frac{1}{U} \sum_{i}\left\{\Delta_{i}\left(\frac{\partial}{\partial \lambda^{i}}\right)^{2}+\varepsilon_{i}\left[V^{r}{ }_{i} \frac{\partial}{\partial \varphi^{2 r}}\right]^{2}\right\}
$$


while the covariant metric tensor takes the slightly more complicated form

$$
d s^{2}=U \sum_{i}\left\{\frac{\left(d \lambda^{i}\right)^{2}}{\Delta_{i}}+\frac{\varepsilon_{-i}}{V^{2}}\left[(r) V^{-r_{i}} d \varphi^{2 r}\right]^{2}\right\},
$$

where we have introduced the determinant

$$
V=\operatorname{det}\left(V^{r}{ }_{i}\right)
$$

and used the fact that the inverse matrix $V_{r}{ }^{i}$ of $V^{r}{ }_{i}$ is given by

$$
V_{r}^{i}=\frac{(i r) V^{-r}-i}{V} \text {. }
$$

At this stage it would still be possible to simplify the canonical expressions (57) and (58) by variable co-ordinate scale changes so as to reduce the functions $\Delta_{i}$ to the values \pm 1 .

The forms of the metric expressions (57) and (58) show that condition IV implies the existence of a natural orthonormal tetrad, which in general is canonically determined in a unique way by the separation. The determination will be ambiguous only in the case where transfers of constants between the functions $W_{1}^{r s}, W_{-1}^{r s}$ can be made in such a way as to preserve the conditions (54); this will usually be impossible, but there exist special cases when the pairs of functions $V^{1}{ }_{i}, V^{-1}{ }_{i}$ are functionally related in a certain well defined manner.

When charge is present under the above conditions the HamiltonJacobi equation (42) reduces to the form

$$
-\bar{m}^{2} U=\sum_{i}\left\{\Delta_{i} S_{i}^{\prime 2}+\varepsilon_{i}\left[V_{i}^{r} \Phi_{2 r}-\bar{e} V_{i}^{r} A_{2 r}\right]^{2} .\right.
$$

It is obvious that condition IV will be satisfied for all values of the charge if $V{ }_{i} A_{2}$ has the form

$$
V^{r}{ }_{i} A_{2 r}=\Xi_{i}\left(\lambda^{i}\right)
$$

and it can easily be checked that it cannot hold in any other circumstances. This is equivalent to satisfying the system (51), (52), (53) by setting

$$
Y_{i}^{s_{i}}=\varepsilon_{i} V_{i}^{s_{i}} \Xi_{i},
$$

which demonstrates that condition IV does indeed make possible a non trivial solution. By (60), the potential components themselves take the form

$$
A_{2 r}=\frac{1}{V} \sum_{i}(i r) V^{-r}{ }_{-i} \Xi_{i} .
$$

Thus when conditions I to IV are satisfied the metric must be reducible to the form (58) which depends on the eight arbitrary single variable functions $U_{i}, V^{r}{ }_{i}, \Delta_{i}$ (of which the last pair is really redundant) and the Maxwell field is determined by (49) and (64) which involve the two further arbitrary single variable functions $\Xi_{i}$. 
By determining a canonical orthonormal tetrad, condition IV reduces the system to a form in which it is comparatively simple to calculate the curvature and Einstein tensors, as will in fact be done in the next section. Nevertheless, in order to obtain the Einstein and Einstein-Maxwell equations in a form which is easily soluble, some further simplifying restriction is desirable. A suitable restriction is obtained in a very convenient manner by requiring that the separability condition (III) be strengthened to apply to the Schrodinger equation (16):

(IIIS). The Schrodinger equation is separable in a manner analogous to that required for the Hamilton-Jacobi equation by condition III.

We shall first show that (IIIS) is indeed a strengthened form of (III) i.e. that (III) automatically holds when (IIIS) does.

Since $\Psi$ is analogous to $e^{-i S}$ rather than to $S$ itself, condition (IIIS) demands that the solution be expressed as a product of functions each depending on one variable only, not as a sum. As in the previous case the dependence of the solution on affine distance and the ignorable coordinates can be seen as once, so we obtain the form

$$
\Psi=e^{i\left(\frac{1}{2} \bar{m}^{2} \tau-\Phi_{2 r} \varphi^{2} r\right)} \prod_{i} \Psi_{i}\left(\lambda^{i}\right)
$$

as the analogue of (41). When this form is substituted, the Schrodinger equation reduces to

$$
\begin{aligned}
-\bar{m}^{2} U= & -\sum_{i} U g^{i j} \frac{\Psi_{i}^{\prime \prime}}{\Psi_{i}}-\sum_{i \neq j} U g^{i j} \frac{\Psi_{i}^{\prime} \Psi_{i}^{\prime}}{\Psi_{i} \Psi_{j}} \\
& -\sum_{i, j} U\left(\frac{\Psi_{i}^{\prime}}{\Psi_{j}}+i \bar{e} A_{j}\right)|g|^{-1 / 2} \frac{\partial}{\partial \lambda^{i}}\left(|g|^{1 / 2} g^{i j}\right) \\
& -\sum_{i, j} U g^{i j}\left(2 i \bar{e} A_{j} \frac{\Psi_{i}^{\prime}}{\Psi_{i}}+i \bar{e} \frac{\partial A_{j}}{\partial \lambda^{j}}-\bar{e}^{2} A_{i} A_{j}\right) \\
& +U g^{2 r} \mathbf{2 s}\left(\Phi_{2 r}-\bar{e} A_{2 r}\right)\left(\Phi_{2 s}-\bar{e} A_{2 s}\right)
\end{aligned}
$$

as the analogue of (42). By reasoning precisely analogous to that used in the Hamilton-Jacobi case we can check one by one that the conditions (43) to (47) and (49) to (53) are necessary in this case, thus confirming that (III) is indeed a consequence of (IIIS).

However, Eqs. (43) to (47) and (49) to (53) are not sufficient in the Schrodinger case. By Eqs. (43) to (47) we have

$$
g=\frac{U^{4}}{W \prod_{i} \Delta_{i}}
$$

where we have introduced the abbreviation

$$
W=\operatorname{det}\left(W^{r s}\right)
$$


and on substitution of all these conditions, (66) reduces to

$$
\begin{aligned}
-\bar{m}^{2} U= & -\sum_{i} \Delta_{i}\left\{\frac{\Psi_{i}^{\prime \prime}}{\Psi_{i}}-\frac{1}{2} \frac{\Psi_{i}^{\prime}}{\Psi_{i}} \frac{\partial}{\partial \lambda^{i}}\left[\ln \frac{U^{2} \Delta_{i}}{W}\right]\right\} \\
& +W^{r s} \Phi_{2 r} \Phi_{2 s}-2 \bar{e} Y^{r} \Phi_{2 r}+\bar{e}^{2} \Omega .
\end{aligned}
$$

It is clear that the only additional requirement to be satisfied is that the co-efficient of $\Psi_{i}^{\prime}$ shall be a function of $\lambda^{i}$ only, and it is obvious that a necessary and sufficient condition for this is that the quantity $U^{2} \Delta_{i} / W$ inside the logarithm should consist of the product of a function of $\lambda^{i}$ only and of a function independent of $\lambda^{i}$. This condition will be satisfied for both values of $i$ if and only if the quantity $U^{2} / W$ is itself a product of two functions each depending on only one of the non-ignorable coordinates. It is convenient at this point to make use of the freedom to adjust the functions $\Delta_{i}$ by a form preserving co-ordinate change so that they co-incide (except possibly in sign) with the two functions so defined. Thus we may set

$$
U^{2}=\varepsilon W \Pi_{i} \Delta_{i},
$$

where $\varepsilon$ is an indicator taking one of the values \pm 1 in order to allow for the fact that the signs of the quantities $\Delta_{i}$ cannot be altered. Condition (70) determines each of the quantities $\Delta_{i}$ to within a constant factor.

Thus conditions (I), (II) and (IIIS) will be satisfied if and only if the metric can be reduced to the form (48) subject, not only to (43) and (47) as before, but in addition to the much more stringent restriction (70) while the Maxwell field is given by the system (49) to (53) as before.

It is clear that condition (IV) has exactly the same content when applied in conjunction with (IIIS) as it does in conjunction with (III), i.e. it is equivalent to (54) and (62). Since Eq. (56) implies

$$
W=V^{2} \prod_{i} \varepsilon_{i}
$$

it follows that when condition (IV) holds the additional condition (70) for (IIIS) can be written in the form

$$
U=-V \prod_{i}\left|\Delta_{i}\right|^{1 / 2}
$$

[By choosing the appropriate signs for the quantities $V_{i}^{r}$ which were left ambiguous by the defining Eq. (56), we can ignore the ambiguity of sign which would otherwise result from taking the square root of (70).]

In order to obtain condition (70) in a simpler form, it is convenient to make a further rescaling. We shall replace the quantities $V^{r}$ and $\Xi_{i}$ by

and

$$
Z^{r}{ }_{i}=V^{-r_{i}}\left|\Delta_{i}\right|^{1 / 2}
$$

$$
X_{i}=(i) \Xi_{i}\left|\Delta_{i}\right|^{1 / 2} \text {. }
$$


In consequence of the former we obtain

where

$$
Z=-\underset{i}{V}\left|\Delta_{i}\right|^{1 / 2}
$$

$$
Z=\operatorname{det}\left(Z^{r}{ }_{i}\right)
$$

At this point we explicitly introduce the condition that the metric has the Lorentz (i.e. hyperbolic normal) signature of ordinary space, which enables us to absorb the unknown signs of the functions $\Delta_{i}$ (which determine $\varepsilon$ ) with the indicators $\varepsilon$, using the freedom to permute the labels \pm 1 of the non-ignorable co-ordinates.

Thus we deduce that it is necessary and sufficient for the conditions (I), (II), (III), (IV) to be satisfied that (by (57)) the covariant metric tensor can be reduced to the canonical form

$$
d s^{2}=\sum_{i} U\left\{\frac{\left(d \lambda^{i}\right)^{2}}{\Delta_{i}}+(i) \frac{\Delta_{i}}{Z^{2}}\left[(r) Z_{i}{ }_{i} d \varphi^{2 r}\right]^{2}\right\}
$$

while (by (59) and (64)) the covariant vector potential can be reduced to the canonical form

$$
A=\sum_{i} \frac{X_{-i}}{Z}\left[(r) Z_{i}^{r} d \varphi^{2 r}\right]
$$

where, so far we can make an arbitrary choice of the eight single variable functions $U_{i}, Z^{r}{ }_{i}, \Delta_{i}$ (two of which are redundant) determining the metric and the two single variable functions $X_{i}$ determining the field. In this canonical system the additional necessary and sufficient condition (54) for Schrodinger separability, (IIIS), takes the very simple form

$$
U=Z \text {. }
$$

This is a fairly strong restriction : although it removes the redundancy, it also has the effect (as we shall see in section 5) of allowing only two of the six functions $U_{i}, Z^{r}$ i to be given independently, so that the metric is limited to four instead of effectively six independent arbitrary single variable functions.

For future reference, we give the inverse metric to (77) as

$$
\left(\frac{\partial}{\partial s}\right)^{2}=\frac{1}{U} \sum_{i}\left\{\Delta_{i}\left(\frac{\partial}{\partial \lambda^{i}}\right)^{2}-\frac{(i)}{\Delta_{i}}\left[Z^{-r} i \frac{\partial}{\partial \varphi^{2 r}}\right]^{2}\right\}
$$

The remainder of this paper will be entirely devoted to a study of the canonical forms (77), (78). We shall work with the general (HamiltonJacobi) form as long as possible, in far't throughout the next section. The Schrodinger condition (79) will be invoked later on in section 5 when further simplification becomes desirable. 


\section{The Hamilton-Jacobi Separable Case}

At this point it is convenient to specify the signiture of the metric completely. Without loss of generality we can restrict our attention to the case where it is +2 , which is equivalent to demanding that the quantity $U \Delta_{-1}$ in (77) shall be positive.

In computing the curvature components in this and the subsequent sections we shall make the further assumption that $U \Delta_{1}$ is positive. This does involve a loss of generality in the short run - it is equivalent to considering only the case where the surfaces of transitivity are timelike (as will be the case for a metric which is axisymmetric and stationary in the strict sense) - but when the algebraic forms of the components have been calculated they will be valid independently of this condition.

We shall use the notation $\omega^{\mu}$, where $\mu$ runs over $\pm 1, \pm 2$, for the differential forms of the canonical orthonormal tetrad: thus subject to the above assumptions we may define

$$
\begin{gathered}
\omega^{i}=\left(\frac{U}{\Delta_{i}}\right)^{1 / 2} d \lambda^{i}, \\
\omega^{2 i}=\frac{\left(U \Delta_{-i}\right)^{1 / 2}}{Z}\left[(r) Z_{i}^{r} d \varphi^{2 r}\right],
\end{gathered}
$$

so that the covariant metric tensor (78) takes the form

$$
d s^{2}=\sum_{i}\left\{\left(\omega^{i}\right)^{2}+(i)\left(\omega^{2 i}\right)^{2}\right\}
$$

while the covariant vector potential (78) takes the form

$$
A=\sum_{i} \frac{X_{-i}}{\left(U \Delta_{-i}\right)^{1 / 2}} \omega^{2 i}
$$

This mode of expression of the metric and field provides a very convenient starting point for the calculation of the quantities we shall need using the exterior calculus, which will obviate the need to work out large numbers of Christoffel symbols, etc. In the following work, the later Greek letters, $\mu, v, \varrho, \sigma$ will always refer to tetrad (not co-ordinate) indices running over $\pm 1, \pm 2$, and they will be raised and lowered using the tetrad components of the metric, determined by (83) from the definition

$$
d s^{2}=g_{\mu \nu} \omega^{\mu} \omega^{\nu} .
$$

The tetrad components of the electromagnetic field can be read off from the formula

$$
F=F_{\mu \nu} \omega^{\mu} \wedge \omega^{\nu}
$$

where $F$ has been calculated from (84) using (25), and hence the components of the electromagnetic energy tensor can be obtained from the formula

$$
4 \pi T_{\mu \nu}=F_{\mu}{ }^{\varrho} F_{\nu \varrho}-\frac{1}{4} F_{\varrho}{ }^{\sigma} F^{\varrho}{ }_{\sigma} g_{\mu \nu},
$$


while the current components can be read off from

where

$$
j=j_{\mu} \omega^{\mu}
$$

$$
4 \pi j=3 * d^{*} F
$$

and where an asterisk denotes the Hodge orthogonal conjugation (i.e. dual) operator. Following Misner (1964) we can take advantage of the fact (see e.g. WILMORE, 1950) that the connection components are determined uniquely by the conditions

and

$$
d \omega^{\mu}=-\omega^{\mu}{ }_{\nu} \wedge \omega^{\mu}
$$

$$
\omega_{\mu \nu}+\omega_{v \mu}=0,
$$

where the latter equation holds in consequence of the fact that the metric components in (85) are constant. Once the connection components have been found, the curvature form can be calculated directly as

$$
\theta^{\mu}{ }_{\nu}=d \omega^{\mu}{ }_{\nu}+\omega^{\mu}{ }_{Q} \wedge \omega^{\varrho}
$$

and the Riemann tensor components can then be read out from the formula

The Ricci form

$$
\theta^{\mu}{ }_{\nu}=\frac{1}{2} R^{\mu}{ }_{\nu \varrho \sigma} \omega^{\varrho} \wedge \omega^{\sigma} .
$$

is then defined by

$$
\theta=R_{u \nu} \omega^{\mu} \omega^{\nu}
$$

$$
R_{\mu \nu}=R_{\mu \varrho \nu}{ }_{\mu \nu} .
$$

The components of the Weyl conformal tensor can be read out from the formula

$$
\Omega^{\mu}{ }_{\nu}=\frac{1}{2} C^{\mu}{ }_{\nu \varrho \sigma} \omega^{\varrho} \wedge \omega^{\sigma}
$$

where we define the Weyl form (cf. e.g. Schouten, 1954) by

$$
\Omega^{\mu \nu}=\theta^{\mu \nu}+R^{[\mu}{ }_{\varrho} \omega^{\nu]} \wedge \omega^{\varrho}+\frac{1}{6} R \omega^{\mu} \wedge \omega^{\nu}
$$

(using square brackets to denote antisymmetrization) and where

$$
R=R_{\mu}{ }^{\mu}
$$

is the Ricci scalar. Finally the Einstein tensor components are defined by

$$
G_{\mu \nu}=R_{\mu \nu}-\frac{1}{2} R g_{\mu \nu}
$$

so that in the presence of a $\Lambda$ term the Einstein equations take the form

$$
G_{\mu \nu}=8 \pi T_{\mu \nu}+\Lambda g_{\mu \nu} .
$$


In order to present the results of these calculations concisely, we introduce the following abbreviations:

together with

$$
\begin{array}{lll}
\alpha_{i}=\frac{\Delta_{i}}{U} & \tilde{\alpha}_{i}=\frac{\Delta_{i}^{\prime}}{U} & \tilde{\alpha}_{i}=\frac{\Delta_{i}^{\prime \prime}}{U} \\
\xi_{i}=\frac{X_{2}}{U} & \tilde{\xi}_{i}=\frac{X_{i}^{\prime}}{U} & \tilde{\xi}_{i}=\frac{X_{i}^{\prime \prime}}{U}
\end{array}
$$

$$
\begin{array}{cc}
\tilde{\eta}_{i}=\frac{1}{2} \frac{U_{i}^{\prime}}{U} & \tilde{\eta}_{i}=\frac{1}{2} \frac{U_{i}^{\prime \prime}}{U} \\
\tilde{\gamma}_{i}=\frac{(i r)}{2} \frac{Z^{-r}-i Z_{i}^{r_{i}^{\prime}}}{Z} & \widetilde{\gamma}_{i}=\frac{(i r)}{2} \frac{Z^{-r}{ }_{-i} Z_{i}^{r^{\prime \prime}}}{Z} \\
\tilde{\beta}_{i}=\frac{(i r)}{2} \frac{Z_{i}^{r} Z^{-r_{i}^{\prime}}}{Z} & \widetilde{\beta}_{i}=\frac{(i r)}{2} \frac{Z_{i}^{r_{i}} Z^{-r_{i}^{\prime \prime}}}{Z} .
\end{array}
$$

The results of the calculations can now be stated as follows. By (25) and (84) we have

where

$$
F=2 \sum_{i} E_{i} \omega^{i} \wedge \omega^{-2 i}
$$

and hence, by (87)

$$
E_{i}=\tilde{\xi}_{i}-2 \tilde{\gamma}_{i} \tilde{\xi}_{i}+2 \tilde{\beta}_{i} \tilde{\xi}_{-i}
$$

and, by (89),

$$
8 \pi T_{\mu \nu}=\left(\sum_{i} E_{i}^{2}\right) \sum_{j}\left(\delta_{\mu}^{2 j} \delta_{\nu}^{2 j}-(j) \delta_{\mu h}^{j} \delta_{v}^{j}\right)
$$

$$
\begin{aligned}
4 \pi j= & \sum_{i}\left(\alpha_{i}\right)^{1 / 2}\left[\widetilde{\xi}_{i}-2 \tilde{\gamma}_{i} \tilde{\xi}_{+}+2 \tilde{\beta}_{-i} \tilde{\xi}_{-i}-2\left(\gamma_{i}-2 \tilde{\gamma}_{i}^{2}-2 \tilde{\beta}_{-i}^{2}\right) \xi_{i}\right. \\
& \left.+2\left(\widetilde{\beta}_{i}-2 \tilde{\beta}_{i} \tilde{\gamma}_{i}-2 \tilde{\beta}_{-i} \tilde{\gamma}_{-i}\right) \xi_{-i}\right] \omega^{-2 i} .
\end{aligned}
$$

Differentiation of Eq. (82) and (81) gives

$$
\begin{aligned}
d \omega^{2 i}= & \left(\alpha_{i}\right)^{1 / 2} \tilde{\eta}_{i} \omega^{i} \wedge \omega^{2 i}+\left[\left(\alpha_{-i}\right)^{1 / 2}\left(\tilde{\eta}_{-i}-2 \tilde{\gamma}_{-i}\right)\right. \\
& \left.+\frac{1}{2}\left(\alpha_{i}\right)^{-1 / 2} \tilde{\alpha}_{i}\right] \omega^{-i} \wedge \omega^{2 i}+2\left(\alpha_{i}\right)^{1 / 2} \tilde{\beta}_{i} \omega^{i} \wedge \omega^{-2 i}, \\
d \omega^{i}= & \left(\alpha_{-i}\right)^{1 / 2} \tilde{\eta}_{-i} \omega^{-i} \wedge \omega^{i},
\end{aligned}
$$

from which it can be checked that the solution of the system (90), (91) is

$$
\begin{aligned}
\omega^{2 i}{ }_{i} & =\left(\alpha_{i}\right)^{1 / 2} \tilde{\eta}_{i} \omega^{2 i}+\left(\alpha_{-i}\right)^{1 / 2} \tilde{\beta}_{i} \omega^{-2 i}, \\
\omega^{2 i}{ }_{-i}= & {\left[\left(\alpha_{-i}\right)^{1 / 2}\left(\tilde{\eta}_{-i}-2 \tilde{\gamma}_{-i}\right)+\frac{1}{2}\left(\alpha_{i}\right)^{-1 / 2} \tilde{\alpha}_{i}\right] \omega^{2 i}-\left(\alpha_{i}\right)^{1 / 2} \tilde{\beta}_{-i} \omega^{-2 i}, } \\
\omega^{2 i}{ }_{-2 i} i & =-\sum_{j}\left(\alpha_{-j}\right)^{1 / 2} \tilde{\beta}_{j} \omega^{j}, \\
\omega_{-i}^{i} & =\sum_{j}(i j)\left(\alpha_{-j}\right)^{1 / 2} \tilde{\eta}_{-j} \omega^{j},
\end{aligned}
$$

where the remaining components are determined from these immediately by the antisymmetry (91). From the symmetry conditions (I) and (II), 
together with the ordinary Reimann tensor identities, it follows that the curvature forms must have the algebraic structure

$$
\begin{gathered}
\theta_{-i}^{i}=-H \omega^{i} \wedge \omega^{-i}+I \omega^{2 i} \wedge \omega^{-2 i}, \\
\theta_{-2 i}^{2 i}=(i) I \omega^{i} \wedge \omega^{-i}+J \omega^{2 i} \wedge \omega^{-2 i} \\
\theta_{-i}^{2 i}=L_{i} \omega^{i} \wedge \omega^{2 i}+N_{i} \omega^{-i} \wedge \omega^{2 i}+B_{i} \omega^{i} \wedge \omega^{-2 i}+(i) K \omega^{-i} \wedge \omega^{-2 i}, \\
\theta_{-i}^{2 i}=N_{i} \omega^{i} \wedge \omega^{2 i}+M_{-i} \omega^{-i} \wedge \omega^{2 i}+(i)(I+K) \omega^{i} \wedge \omega^{-2 i}- \\
-B_{-i} \omega^{-i} \wedge \omega^{-2 i}
\end{gathered}
$$

where the remaining components are determined from these by the antisymmetry of $\theta^{\mu \nu}$. Straightforward computation from (112) to (115) according to (92) yields the specific values:

$$
\begin{gathered}
H=\sum_{j}\left[\frac{1}{2} \tilde{\alpha}_{j} \tilde{\eta}_{j}+\alpha_{j}\left(\eta_{j}-2 \tilde{\eta}_{j}^{2}\right)\right] \\
I=\sum_{j}(j) \tilde{\beta}_{j}\left(\frac{1}{2} \tilde{\alpha}_{-j}-2 \alpha_{-j} \tilde{\gamma}_{-j}\right) \\
J=\sum_{j}\left[\frac{1}{2} \tilde{\alpha}_{j} \tilde{\eta}_{j}+\alpha_{j}\left(\tilde{\eta}_{j}^{2}-2 \tilde{\eta}_{j} \tilde{\gamma}_{j}+\tilde{\beta}_{-j}^{2}\right)\right] \\
K=\sum_{j}(j) \tilde{\beta}_{j}\left(\frac{1}{2} \tilde{\alpha}_{-j}-2 \alpha_{-j} \tilde{\gamma}_{-j}\right) \\
L_{i}=\frac{1}{2} \tilde{\alpha}_{i} \tilde{\eta}_{i}+\frac{1}{2} \tilde{\alpha}_{-i} \tilde{\eta}_{-i}+\alpha_{i}\left(\tilde{\eta}_{i}-2 \tilde{\eta}_{i}^{2}\right)+\alpha_{-i}\left(\tilde{\eta}_{-i}^{2}-2 \tilde{\eta}_{-i} \tilde{\gamma}_{-i}+\tilde{\beta}_{i}^{2}\right) \\
M_{i}=\frac{1}{2} \tilde{\alpha}_{i}+\tilde{\alpha}_{i}\left(\tilde{\eta}_{i}-3 \tilde{\gamma}_{i}\right)+\alpha_{i}\left(\tilde{\eta}_{i}-2 \tilde{\gamma}_{i}-2 \tilde{\eta}_{i}^{2}-2 \tilde{\eta}_{i} \tilde{\gamma}_{i}+8 \tilde{\gamma}_{i}^{2}\right) \\
+\alpha_{-i}\left(\tilde{\eta}_{-i}^{2}-3 \tilde{\beta}_{i}^{2}\right), \\
N_{i}=3\left(\alpha_{i} \alpha_{-i}\right)^{1 / 2}\left(\tilde{\beta}_{i} \tilde{\beta}_{-i}-\tilde{\eta}_{i} \tilde{\eta}_{-i}\right) \\
B_{i}=\left(\alpha_{i} \alpha_{-i}\right)^{1 / 2}\left(\tilde{\beta}_{i}+\tilde{\beta}_{i}\left(\tilde{\eta}_{i}-2 \tilde{\gamma}_{i}\right)-\tilde{\beta}_{-i} \tilde{\eta}_{-i}\right)
\end{gathered}
$$

It is apparent that these twelve quantities are not independent, but are connected by the three identies

and

$$
\begin{aligned}
K & =I, \\
H+J & =\sum_{i} L_{i}
\end{aligned}
$$

$$
N_{i}=N
$$

say, (i.e. $N_{i}$ is independent of $i$ ).

It may also be observed at this point that the curvature components contain only positive powers of the quantities defined in (101) to (105). Since the only factors appearing in the denominators of these quantities are $U$ and $Z$ we see that the Riemann tensor is well behaved except 
where one of these vanishes, which suggests that the other singularities of the metric form (77), which occur where either of the functions $\Delta_{\lambda}, \Delta_{\mu}$ vanishes, need not represent geometric singularities but only failures of the canonical co-ordinate system.

In terms of the functions which have just been defined, and after simplifications using the identies (128), (129), (130), the Ricci form is obtained as

$$
\begin{gathered}
\theta=-\sum_{i}\left\{\left(H+L_{i}+M_{i}\right)\left(\omega^{i}\right)^{2}+(i)\left(J+L_{i}+M_{-i}\right)\left(\omega^{2 i}\right)^{2}\right. \\
\left.+2 N \omega^{i} \omega^{-i}+\left(\sum_{j}(j) B_{-j}\right) \omega^{2 i} \omega^{-2 i}\right\}
\end{gathered}
$$

and hence we obtain the Ricci scalar as

$$
R=-2 \sum_{j}\left(2 L_{j}+M_{j}\right)
$$

and the Weyl form components as

$$
\begin{gathered}
\Omega_{-i}^{i}=-\frac{1}{6} \sum_{j}\left(L_{j}-M_{j}\right) \omega^{i} \wedge \omega^{-i}+I \omega^{2 i} \wedge \omega^{-2 i} \\
\Omega_{-2 i}^{2 i}=(i) I \omega^{i} \wedge \omega^{-i}+\frac{(i)}{6} \sum_{j}\left(L_{j}-M_{j}\right) \omega^{2 i} \wedge \omega^{-2 i} \\
\Omega_{i}^{2 i}=\frac{1}{6} \sum_{j}\left(L_{j}-M_{j}\right) \omega^{i} \wedge \omega^{2 i}+(i) I \omega^{-i} \wedge \omega^{-2 i}+\frac{1}{2} \sum_{i} B_{j} \omega^{i} \wedge \omega^{-2 i}, \\
\Omega_{-i}^{2 i}=2(i) I \omega^{i} \wedge \omega^{-2 i}+\frac{1}{3} \sum_{j}\left(L_{j}-M_{j}\right) \omega^{-i} \wedge \omega^{2 i}-\frac{1}{2} \sum_{j} B_{j} \omega^{-i} \wedge \omega^{-2 i},
\end{gathered}
$$

where the remaining components are determined from these by the antisymmetry of $\Omega^{\mu \nu}$.

It can be seen immediately from this array that the canonical tetrad defined by the separability condition (IV) will also be the canonical tetrad of the Weyl tensor in the Petrov-Pirani classification (see e.g. EHLERs [10]) provided that the condition

$$
\sum_{j} B_{j}=0
$$

is satisfied (but not otherwise). Moreover, whenever this condition does hold, the conformal tensor will be of type D (except in the case where it vanishes altogether) and the two principle null forms will be

$$
\omega^{1} \pm \omega^{-2} \text {. }
$$

In terms of the canonical co-ordinates (and after adjusting the normalisation in order to give maximum simplicity) the two principle null vectors will be expressiable in contravariant form as

$$
\frac{\partial}{\partial \lambda^{1}} \pm\left[Z^{-r}{ }_{1} \frac{\partial}{\partial \varphi^{2 r}}\right] \text {. }
$$


By Eq. (99), (131), (132), the Einstein tensor components are given by

$$
\begin{aligned}
G_{2 i, j} & =0, \\
G_{1,-1} & =-2 N, \\
G_{2,-2} & =-\sum_{j}(j) B_{j}, \\
G_{i, i} & =J+L_{-i}+M_{-i}, \\
G_{2 i, 2 i} & =(i)\left(H+L_{-i}+M_{i}\right) .
\end{aligned}
$$

On substituting the explicit formulae (120) to (127) into (141) and (142) we obtain for the off diagonal components which do not identically vanish

$$
\begin{aligned}
& G_{1,-1}=6\left(\alpha_{1} \alpha_{-1}\right)^{1 / 2}\left(\eta_{1} \eta_{-1}-\beta_{1} \beta_{-1}\right), \\
& G_{2,-2}=-\left(\alpha_{1} \alpha_{-1}\right)^{1 / 2} \sum_{j}(j)\left[\tilde{\beta}_{j}+2 \tilde{\beta}_{j}\left(\tilde{\eta}_{j}-\tilde{\gamma}_{j}\right)\right] .
\end{aligned}
$$

The analogous expressions obtained for the diagonal components from the pairs (143) and (144) are rather long, and it turns out that the relevant information can be expressed much more simply in terms of the linear combinations

$$
\begin{aligned}
\sum_{i} G_{j j} & =2 J+\sum_{j}\left(L_{j}+M_{j}\right), \\
\sum_{j}(j) G_{j j} & =-\sum_{j}(j)\left(L_{j}+M_{j}\right), \\
G_{-2 i-2 i}+(i) G_{i i} & =(i)(J-H)-\sum_{j}(j) L_{j} .
\end{aligned}
$$

Thus the diagonal components are determined by

$$
\begin{array}{rr}
\sum_{j} G_{j j}=\sum_{j}\left[\frac{1}{2} \tilde{\alpha}_{j}+3 \tilde{\alpha}_{j}\left(\tilde{\eta}_{j}-\tilde{\gamma}_{j}\right)+2 \alpha_{j}\left(\tilde{\eta}_{j}-\tilde{\gamma}_{j}-4 \tilde{\gamma}_{j}\left(\tilde{\eta}_{j}-\tilde{\gamma}_{j}\right)\right)\right] & (150) \\
\sum_{j}(j) G_{j j}=-\sum_{j}(j)\left[\frac{1}{2} \tilde{\alpha}_{j}+\tilde{\alpha}_{j}\left(\tilde{\eta}_{j}-3 \tilde{\gamma}_{j}\right)+2 \alpha_{j}\left(\tilde{\eta}_{j}-\tilde{\gamma}_{j}-3 \tilde{\eta}_{j}^{2}+4 \tilde{\gamma}_{j}^{2}+\tilde{\beta}_{-j}^{2}\right)\right] \\
G_{-2 i-2 i}+(i) G_{i, i}=-2(i) \alpha_{i}\left(\tilde{\eta}_{i}-3 \tilde{\eta}_{i}^{2}+2 \tilde{\eta}_{i} \tilde{\gamma}_{i}-\tilde{\beta}_{-i}^{2}\right) . & (151)
\end{array}
$$

Not only do the formulae for the components take a comparatively simple form in terms of these particular linear combinations: the same applies to the Einstein-Maxwell equations. It follows directly from the symmetry conditions I and II that the electromagnetic current has no components orthogonal to the surfaces of transitivity and that the energymomentum and Einstein tensors contain no cross components between directions in and orthogonal to the surfaces of transitivity, as can be verified in Eqs. (108), (109) and (140). Therefore there remain only eight of the source-free Einstein-Maxwell equations which are not satisfied 
identically, and they can be written in the form

$$
\begin{aligned}
G_{1,-1} & =0, \\
G_{2,-2} & =0, \\
\sum_{j} G_{j, j} & =2 \Lambda, \\
\sum_{j}(j) G_{j, j} & =-2 \sum_{j} E_{j}^{2}, \\
G_{2 i 2 i}-(i) G_{-i,-i} & =0, \\
j_{2 i} & =0 .
\end{aligned}
$$

In terms of the quantities defined by (107) and (120) to (127) this system can be reformulated as

$$
\begin{aligned}
N & =0, \\
B_{1} & =B_{-1}, \\
M_{1}+M_{-1} & =2(\Lambda-2 H), \\
M_{1}-M_{-1} & =2\left(E_{1}^{2}+E_{-1}^{2}\right), \\
J_{i} & =H, \\
L_{i} & =H,
\end{aligned}
$$

together with (158), and where the three equations of the subsystem (163), (164) are not independent but include the identity (129). The simplest form of the expanded system is obtained by substituting (145), (146) and (150) to (152) directly into (153) to (157), but unfortunately despite the strength of the separability conditions which have been imposed so far the system is still more complicated than could be desired. Therefore in order to make further progress we shall now impose the Schrodinger separability condition (79).

\section{The Schrodinger Separable Case}

When written out in full the Schrodinger separability condition (29) takes the form

$$
U_{\varepsilon}+U_{-\varepsilon}=(\varepsilon)\left(P_{\varepsilon} Q_{-\varepsilon}-P_{-\varepsilon} Q_{\varepsilon}\right)
$$

where $\varepsilon$ has a fixed value in the range $( \pm 1)$ of the variable $(i)$ and where we have introduced the notation

$$
P_{i}=Z^{1}{ }_{i}, \quad Q_{i}=Z^{-1}{ }_{i}
$$

in order to distinguish the functions $Z^{ \pm 1}{ }_{i}$ more clearly since they will no longer be treated in the same way. Bearing in mind that each of the functions in (165) depends only on the variable denoted by its suffix, we see that there are just three essentially different ways in which this equation can be satisfied. 
Case (a). If neither function $U_{i}$ is constant then two of the factors on the right hand side of (165) must be constant, one in each variable and one in each term. By swapping the labels of the co-ordinates $\varphi^{2 r}$ if necessary we can arrange without loss of generality that these functions are $Q_{\varepsilon}$ and $Q_{-\varepsilon}$, and by suitable co-ordinate scale changes we can arrange that these constant values are both unity. Finally, using the freedom to transfer a constant between the terms $U_{i}$ we can obtain the canonical form

$$
[a]\left\{\begin{array}{l}
U_{i}=(i) P_{i} \\
Q_{i}=1
\end{array}\right.
$$

If one of the functions $U_{i}$ is constant, it can be made to vanish by adjusting the values of the other, and moreover under these circumstances one of the terms on the right hand side of (165) can be made to vanish also. (If neither of these terms vanishes to begin with, the factors $P_{i}, Q_{i}$ must be proportional for one or other value of $i$, and so one of them can be removed by a co-ordinate change in which the co-ordinates $\varphi^{2 r}$ are replaced by linear combinations of themselves with constant coefficients.) There are two ways in which this can happen:

Case (b). If the term on the right hand side of (165) which vanishes does so because of the vanishing of the factor depending on the same variable as the surviving term in $U$, then by suitable co-ordinate scale changes, and by choice of $\varepsilon$, we can obtain the canonical form

$$
[b(\varepsilon)]\left\{\begin{array}{l}
U_{\varepsilon}=(\varepsilon) P_{\varepsilon} \\
U_{\varepsilon}=0 \\
Q_{\varepsilon}=0 \\
Q_{-\varepsilon}=1 .
\end{array}\right.
$$

Case (c). If the term on the right hand side of (165) which vanishes does so because of the vanishing of the factor depending on the same variable as the vanishing term in $U$, then by similar adjustments we can obtain the canonical form

$$
[c(\varepsilon)]\left\{\begin{array}{l}
U_{\varepsilon}=(\varepsilon) P_{\varepsilon} \\
U_{-\varepsilon}=0 \\
P_{-\varepsilon}=0 \\
Q_{-\varepsilon}=0 .
\end{array}\right.
$$

This exhausts the possibilities. In consequence of the arrangement of signs in (77) there is a qualitative difference between the two possible values of $\varepsilon$ in Case (b) and Case (c), so we really have five distinct canonical forms, namely $[a],[b( \pm 1)],[c( \pm 1)]$.

21 Commun.math. Phys., Vol. 10 
Each of these canonical forms depends on just four independent arbitrary single variable functions. In form $[a]$, which is the most symmetric, there are two functions of each variable, namely $\Delta_{ \pm 1}$ and $U_{ \pm 1}$. In the forms $[b(\varepsilon)]$, there are also two functions of each variable, but asymmetrically arranged, namely $\Delta_{ \pm 1}, U_{\varepsilon}$ and $P_{-\varepsilon}$. In the forms $[c(\varepsilon)]$ there is one function of one variable and three of the other: $\Delta_{ \pm 1}, U_{\varepsilon}$ and $Q_{\varepsilon}$.

From (77) we see that the covariant metric tensors for these canonical forms are given by

$$
\begin{aligned}
{[a]: \quad d s^{2}=} & \left(U_{1}+U_{-1}\right)\left\{\sum_{i} \frac{\left(d \lambda^{i}\right)^{2}}{\Delta_{i}}\right\}+\frac{\sum_{i}(i) \Delta_{-i}\left[d \chi-(i) U_{i} d \psi\right]^{2}}{U_{1}+U_{-1}}, \\
{[b(\varepsilon)]: \quad d s^{2}=} & U_{\varepsilon}\left\{\sum_{i} \frac{\left(d \lambda^{i}\right)^{2}}{\Delta_{i}}+(\varepsilon) \Delta_{-\varepsilon} d \psi^{2}\right\} \\
& -(\varepsilon) \frac{\Delta_{\varepsilon}}{U_{\varepsilon}}\left[d \chi-P_{-\varepsilon} d \psi\right]^{2} \\
{[c(\varepsilon)]: \quad d s^{2}=} & U_{\varepsilon}\left\{\sum_{i} \frac{\left(d \lambda^{i}\right)^{2}}{\Delta_{i}}\right\} \\
& +(\varepsilon) \frac{\Delta_{-\varepsilon}\left[Q_{\varepsilon} d \chi_{i}-(\varepsilon) U_{\varepsilon} d \psi\right]^{2}-\Delta_{\varepsilon} d \chi^{2}}{U_{\varepsilon}},
\end{aligned}
$$

where we have introduced the notation $\psi$ and $\chi$ for the adjusted forms of the co-ordinates $\varphi^{2}$ and $\varphi^{-2}$ respectively, since they are no longer being treated on the same footing.

These canonical forms are not mutually exclusive. There are two ways in which they can overlap. If $Q_{k}$ is constant in $[c(\varepsilon)]$ the result is the same as if $U_{-8}$ is constant in $[a]$ since by co-ordinate changes the former can be set equal to unity and the latter to zero. If $Q_{\varepsilon}$ is proportional to $U_{\varepsilon}$ in $[c(\varepsilon)]$ the result is the same as if $P_{-\varepsilon}$ is constant in $[b(\varepsilon)]$ since by co-ordinate changes both can be set equal to zero, the metrics then being diagonal. If both situations occur simultaneously, i.e. if $Q_{\varepsilon}$ is proportional to $U_{\varepsilon}$ and also constant in $[c(\varepsilon)]$ we obtain a degenerate form common to all five of $[a],[b(\varepsilon)]$ and $[c(\varepsilon)]$.

The effects of the canonical conditions $[a],[b(\varepsilon)],[c(\varepsilon)]$ on the functions (103) to (105) can be tabulated as follows:

$$
\begin{aligned}
& {[a]\left\{\begin{array}{l}
\tilde{\eta}_{i}=\tilde{\gamma}_{i}=-\tilde{\beta}_{i}=\frac{1}{2} U_{i}^{\prime} / U \\
\tilde{\eta}_{i}=\tilde{\gamma}_{i}=-\tilde{\beta}_{i}=\frac{1}{2} U_{i}^{\prime \prime} / U
\end{array}\right.}
\end{aligned}
$$

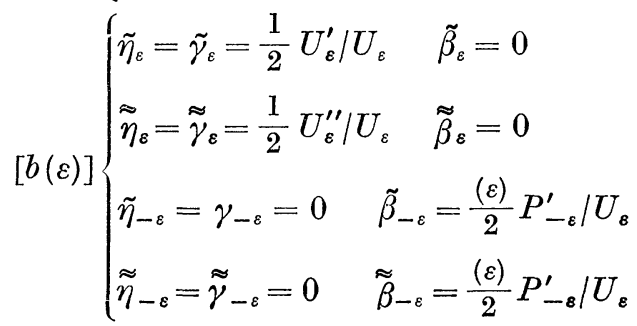




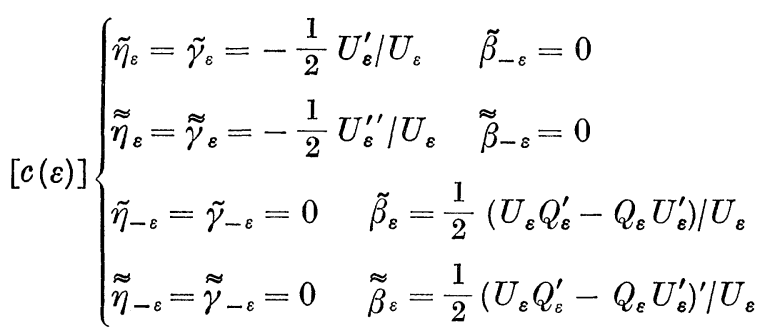

while the functions (101) and (102) are not effectively simplified except that we may replace $U$ by $U_{\varepsilon}$ in $[\mathrm{b}(\varepsilon)]$ and $[c(\varepsilon)]$.

It can be seen that in all cases, although for different reasons, the Eq. (159) (or equivalently (153)) is satisfied as an identity. It can also be seen that in all cases we have

$$
\tilde{\eta}_{i}=\tilde{\gamma}_{i}, \quad \tilde{\eta}_{i}=\tilde{\gamma}_{i} .
$$

Substituting these relations throughout we can expand the remaining seven equations of the Maxwell Einstein system (158) to (164) as

$$
\begin{gathered}
\tilde{\alpha}_{1}+\tilde{\alpha}_{-1}=41, \\
\tilde{\beta}_{1}-\tilde{\beta}_{-1}=0, \\
\tilde{\gamma}_{i}-\tilde{\gamma}_{i}^{2}-\beta_{i}^{2}=0, \\
\sum_{j}(j)\left[2 \alpha_{j} \tilde{\gamma}_{j}-2 \tilde{\alpha}_{j} \tilde{\gamma}_{j}+\frac{1}{2} \tilde{\alpha}_{j}\right]=2 \sum_{j}\left[\xi_{j}-2 \tilde{\gamma}_{j} \tilde{\xi}_{j}+2 \tilde{\beta}_{j} \xi_{-j}\right]^{2}, \\
\tilde{\zeta}_{i}-2 \tilde{\gamma}_{i} \tilde{\xi}_{i}+2 \tilde{\beta}_{-i} \xi_{-i}+2 \tilde{\gamma}_{i} \xi_{i}+2\left(\tilde{\beta}_{i}-2 \tilde{\beta}_{i} \tilde{\gamma}_{i}-2 \tilde{\beta}_{-i} \tilde{\gamma}_{-i}\right) \xi_{-i}=0
\end{gathered}
$$

For further simplification it is necessary to consider the different canonical cases separately. It turns out that in each case the system can be solved completely, the four unknown single variable functions in the metric being obtained as polynomials of not more than fourth degree. The detailed steps in the process of integration in the various cases are somewhat tedious, and therefore we shall not describe them, but will proceed directly to quote the results.

\section{The Source-Free Maxwell Einstein Solutions}

After considerable use of form preserving co-ordinate and gauge transformations, the complete set of solutions of the source-free MaxwellEinstein equations with $\Lambda$ term, having the form (77), (78) and subject 
to (79), can be reduced to the following:

$$
\begin{aligned}
& {[A]\left\{\begin{array}{l}
Z_{i}{ }_{i} \equiv P_{i}=(i) U_{i}=(i)\left(\lambda^{i}\right)^{2} \\
Z^{-1}{ }_{i} \equiv Q_{i}=1 \\
\Delta_{1}=\frac{1}{3} \Lambda\left(\lambda^{1}\right)^{4}+h\left(\lambda^{1}\right)^{2}-2 m_{1} \lambda^{1}+n+e^{2} \\
\Delta_{-1}=\frac{1}{3} \Lambda\left(\lambda^{-1}\right)^{4}-\left[h\left(\lambda^{-1}\right)^{2}-2 m_{-1} \lambda^{-1}-n\right]
\end{array}\right.} \\
& {[B(\varepsilon)]\left\{\begin{array}{l}
Z_{\varepsilon}^{1} \equiv P_{\varepsilon}=(\varepsilon) U_{k}=(\varepsilon)\left[\left(\lambda^{\varepsilon}\right)^{2}+1\right] \\
Z_{\varepsilon}^{-1} \equiv Q_{\varepsilon}=U_{-\varepsilon}=0 \\
Z_{\varepsilon}^{-1} \equiv P_{-\varepsilon}=2 \lambda^{-\varepsilon} \\
Z^{-1}{ }_{-\varepsilon} \equiv Q_{-\varepsilon}=1
\end{array}\right.} \\
& \Delta_{\varepsilon}=\Lambda\left[\frac{1}{3}\left(\lambda^{\varepsilon}\right)^{4}+2\left(\lambda^{\varepsilon}\right)^{2}-1\right]+(\varepsilon)\left[h\left(\left(\lambda^{\varepsilon}\right)^{2}-1\right)\right. \\
& \left.-2 m_{\varepsilon} \lambda^{\varepsilon}+e^{2}\right] \\
& \Delta_{-\varepsilon}=-(\varepsilon)\left[h\left(\lambda^{-\varepsilon}\right)^{2}-2 m_{-k} \lambda^{-\varepsilon}+n\right] \text {. } \\
& {[C(\varepsilon)]\left\{\begin{array}{l}
Z^{1_{\varepsilon}} \equiv P_{\varepsilon}=(\varepsilon) U_{\varepsilon}=(\varepsilon)\left(\lambda^{\varepsilon}\right)^{2} \\
Z^{-1_{\varepsilon}} \equiv Q_{\varepsilon}=0 \\
Z_{-\varepsilon}^{1_{-\varepsilon}} \equiv P_{-\varepsilon}=0 \\
Z_{-\varepsilon}^{-1_{-\varepsilon}} \equiv Q_{-\varepsilon}=1 \\
\Delta_{\varepsilon}=\frac{1}{3} \Lambda\left(\lambda^{\varepsilon}\right)^{4}+(\varepsilon)\left[h\left(\lambda^{\varepsilon}\right)^{2}-2 m_{\varepsilon} \lambda^{\varepsilon}+e^{2}\right] \\
\Delta_{-\varepsilon}=-(\varepsilon)\left[h\left(\lambda^{-\varepsilon}\right)^{2}-2 m_{-\varepsilon} \lambda^{-\varepsilon}+n\right]
\end{array}\right.} \\
& {[D]\left\{\begin{array}{l}
Z^{1}{ }_{i} \equiv P_{i}=(i) U_{i}=\frac{1}{2}(i) \\
Z^{-1}{ }_{i} \equiv Q_{i}=1 \\
\Delta_{i}=\Lambda\left(\lambda^{i}\right)^{2}+(i)\left[e^{2}\left(\lambda^{i}\right)^{2}-2 m_{i} \lambda^{i}+n_{i}\right]
\end{array}\right.}
\end{aligned}
$$

where, in all cases

subject to

$$
\begin{aligned}
& X_{i}=e_{i} \lambda^{i} \\
& e^{2}=\sum_{i} e_{i}^{2}
\end{aligned}
$$

and where apart from this restriction the parameters $\Lambda, e, e_{i}, h, m_{i}, n, n_{i}$ and the co-ordinates $\lambda^{i}$ may take any real values provided the inequality

$$
\Delta_{-1}>0
$$


is satisfied. [If this condition were violated the total signature of the metric would be reversed with the result that the terms containing the factor $e^{2}$ would have the wrong sign.] Variations of the complexion of the electromagnetic field by alteration of the parameters $e_{i}$ subject to (216) with $e^{2}$ constant do not affect the metric: these are the duality rotations, familiar from the already unified field theory of RAINICH, MISNER and WHEELER.

The solutions $[A],[B(\varepsilon)]$, belong to the canonical forms $[a],[b(\varepsilon)]$ respectively. The solution has $[C(\varepsilon)]$ been presented in the terminology of the canonical form $[c(\varepsilon)]$, but it can be seen that it belongs to the restricted class of metrics in which the canonical forms $[b(\varepsilon)]$ and $[c(\varepsilon)]$ overlap. The solution $[D]$ has been presented in the terminology of the canonical form $[a]$ but it belongs to the degenerate class common to all of the forms $[a],[b( \pm 1)],[c( \pm 1)]$.

In all cases the electromagnetic field is such that

$$
\sum_{j} E_{j}^{2}=\frac{e^{2}}{U^{2}}
$$

and therefore by (100) and (108) the Ricci tensor components are

$$
R_{\mu \nu}=\frac{e^{2}}{U^{2}} \sum_{j}\left[\delta_{\mu}^{2 j} \delta_{\nu}^{2 j}-(j) \delta_{\mu}^{j} \delta_{\nu}^{j}\right]-\Lambda g_{\mu \nu}
$$

where the quantity $U$ is given in the various cases as:

$$
\begin{array}{ll}
{[A]:} & U=\left(\lambda^{1}\right)^{2}+\left(\lambda^{-1}\right)^{2}, \\
{[B(\varepsilon)]:} & U=\left(\lambda^{\varepsilon}\right)^{2}+1, \\
{[C(\varepsilon)]:} & U=\left(\lambda^{\varepsilon}\right)^{2}, \\
{[D]: \quad U=1 .}
\end{array}
$$

In all cases also the condition (137) is satisfied, so the Weyl tensor is of type $D$ or zero, its components being

$$
\begin{aligned}
\Omega_{-1}^{i} & =-\left(H-\frac{1}{3} \Lambda\right) \omega^{i} \wedge \omega^{-i}+I \omega^{2 i} \wedge \omega^{-2 i}, \\
\Omega_{-2 i}^{2 i} & =(i) I \omega^{i} \wedge \omega^{-i}+(i)\left(H-\frac{1}{3} \Lambda\right) \omega^{2 i} \wedge \omega^{-2 i}, \\
\Omega^{2 i}{ }_{i} & =\left(H-\frac{1}{3} \Lambda\right) \omega^{i} \wedge \omega^{2 i}+(i) I \omega^{-i} \wedge \omega^{-2 i}, \\
\Omega^{2 i}{ }_{-i} & =2(i) I \omega^{i} \wedge \omega^{-2 i}+2\left(H-\frac{1}{3} \Lambda\right) \omega^{-i} \wedge \omega^{2 i},
\end{aligned}
$$


where the quantities $H-1 / 3 \Lambda$ and $I$ are given as:

$$
\begin{aligned}
& {[A]\left\{\begin{array}{l}
H-\frac{1}{3} \Lambda \\
=\left\{\sum_{j}(j) m_{-j} \lambda^{-j}\left[3\left(\lambda^{j}\right)^{2}-\left(\lambda^{-j}\right)^{2}\right]-e^{2}\left[\left(\lambda^{1}\right)^{2}-\left(\lambda^{-1}\right)^{2}\right]\right\} / U^{3} \\
I=\left\{\sum_{j} m_{j} \lambda^{-j}\left[3\left(\lambda^{j}\right)^{2}-\left(\lambda^{-j}\right)^{2}\right]-2 e^{2} \lambda^{1} \lambda^{-1}\right\} / U^{3} .
\end{array}\right.} \\
& {[B(\varepsilon)]\left\{\begin{array}{c}
H-\frac{1}{3} \Lambda=\left\{\left[\frac{4}{3} \Lambda+(\varepsilon) h\right]\left[3\left(\lambda^{\varepsilon}\right)^{2}-1\right]\right. \\
\left.+(\varepsilon) m_{\varepsilon} \lambda^{\varepsilon}\left[\left(\lambda^{\varepsilon}\right)^{2}-3\right]-(\varepsilon) e^{2}\left[\left(\lambda^{\varepsilon}\right)^{2}-1\right]\right\} / U^{3} \\
I=\left\{\lambda^{\varepsilon}\left[\frac{4}{3} \Lambda+(\varepsilon) h\right]\left[\left(\lambda^{\varepsilon}\right)^{2}-3\right]\right. \\
\left.-(\varepsilon) m_{\varepsilon}\left[3\left(\lambda^{\varepsilon}\right)^{2}-1\right]+2(\varepsilon) e^{2} \lambda^{\varepsilon}\right\} / U^{3}
\end{array}\right.} \\
& {[C(\varepsilon)]\left\{\begin{aligned}
H-\frac{1}{3} \Lambda & =\left\{(\varepsilon) m_{\varepsilon}\left(\lambda^{\varepsilon}\right)^{2}-(\varepsilon) e^{2}\left(\lambda^{\varepsilon}\right)^{2}\right\} / U^{3} \\
I & =0
\end{aligned}\right.} \\
& {[D]\left\{\begin{aligned}
H-\frac{1}{3} \Lambda & =-\frac{1}{3} \Lambda \\
I & =0 .
\end{aligned}\right.}
\end{aligned}
$$

It is apparent from the expressions (219) to (235) that all the spaces except $[D]$ (for which the curvature components are constant) are asymptotically flat as $U \rightarrow \infty$. There are curvature singularities only where $U \rightarrow 0$, a possibility which arises only in the cases $[A]$ and $[C( \pm)]$.

All of the solutions as expressed above contain a considerable number of redundant parameters which could be normalised to \pm 1 or made to vanish by further form preserving co-ordinate changes. We have not performed these operations here because there are many special cases to be considered, the enumeration of which would require much more space. In fact we can perform the reverse process in order to combine the solutions. $[B(+)]$ and $[C(+)]$ in the single expression $[\widetilde{B}(+)]$ given in the introduction by introducing a further redundant parameter $l$ : when $l$ vanishes the form $[C(+)]$ is obtained and in all other cases $l$ can be renormalised to +1 by a co-ordinate change giving the form $[B(+)]$. Similarly by introducing a parameter $k$ we can obtain the expression $[\widetilde{B}(-)]$ combining $[B(-)]$ and $[C(-)]$. With these transformations, and after replacing $\lambda, \mu$ by $\lambda^{1}, \lambda^{-1}$ and the parameters $m, q, n, p$ by $m_{1}, m_{-1}$, $n_{1},-n_{-1}$ respectively, the forms $[A],[\widetilde{B}( \pm)],[D]$, given in the introduction agree exactly with the forms $[A],[B( \pm)],[C( \pm)],[D]$, given immediately above.

By introducing two further redundant co-ordinates $c$ and $\gamma$ one can construct a single form $[\tilde{A}]$ which combines all of $[A],[B( \pm)],[C( \pm)]$, $[D]$. Starting from $[A]$ in the form $(4),(5),(6),(7)$ we make the co- 
ordinate transformation

$$
\begin{aligned}
\tilde{\lambda} & =\frac{\lambda-k}{c \cos \gamma}, \\
\tilde{\mu} & =\frac{\mu-l}{c \sin \gamma}, \\
d \tilde{\psi} & =c \sin \gamma \cos \gamma d \psi, \\
d \tilde{\chi} & =c d \chi+c\left(l^{2} \cos ^{2} \gamma-k^{2} \sin ^{2} \gamma\right) d \psi .
\end{aligned}
$$

We also introduce new parameters $\tilde{h}, \tilde{m}, \tilde{q}, \tilde{n}, \tilde{p}$ in place of $h, m, q, p, e$, defined by

$$
\begin{gathered}
\tilde{h}=h+2 \Lambda\left(k^{2}-l^{2}\right), \\
\tilde{m} c \cos \gamma=m-h k-\frac{2}{3} \Lambda k^{3}, \\
\tilde{q} c \sin \gamma=q-h l+\frac{2}{3} \Lambda l^{3}, \\
\tilde{n} c^{2} \cos ^{2} \gamma=e^{2}+p-2 m k+h k^{2}+\frac{1}{3} \Lambda k^{3}, \\
\tilde{p} c^{2} \sin ^{2} \gamma=p+2 q l-h l^{2}+\frac{1}{3} \Lambda l^{3} .
\end{gathered}
$$

The resulting transformed metric, in which we have dropped the tilda throughout, is

where

$$
\begin{aligned}
d s^{2}= & {\left[(c \lambda \cos \gamma+k)^{2}+(c \mu \sin \gamma+l)^{2}\right]\left\{\frac{d \lambda^{2}}{\Delta_{\lambda}}+\frac{d \mu^{2}}{\Delta_{\mu}}\right\} } \\
& +\frac{\Delta\left\{\sin \gamma d \chi-\left[\left(c^{2} \lambda^{2}+k^{2}+l^{2}\right) \cos \gamma+2 c k \lambda\right] d \psi\right\}^{2}}{(c \lambda \cos \gamma+k)^{2}+(c \mu \sin \gamma+l)^{2}} \\
& -\frac{\Delta\left\{\cos \gamma d \chi+\left[\left(c^{2} \mu^{2}+k^{2}+l^{2}\right) \sin \gamma+2 c l \mu\right] d \psi\right\}^{2}}{(c \lambda \cos \gamma+k)^{2}+(c \mu \sin \gamma+l)^{2}}
\end{aligned}
$$

$$
\begin{aligned}
\Delta_{\lambda}= & \frac{1}{3} \Lambda c^{2} \lambda^{4} \cos ^{2} \gamma+\frac{4}{3} \Lambda c k \lambda^{3} \cos \gamma \\
& +\left(2 \Lambda l^{2}+h\right) \lambda^{2}-2 m \lambda+n \\
\Delta_{\mu}= & \frac{1}{3} \Lambda c^{2} \mu^{4} \sin ^{2} \gamma+\frac{4}{3} \Lambda c l \mu^{3} \sin \gamma \\
& +\left(2 \Lambda k^{2}-h\right) \mu^{2}+2 q \mu+p
\end{aligned}
$$

and the charge parameter $e$ is given in terms of the new parameters by

$$
\begin{aligned}
e^{2}= & \Lambda\left(k^{4}-l^{4}\right)+h\left(k^{2}+l^{2}\right)+2 c(k m \cos \gamma+l q \sin \gamma) \\
& +c^{2}\left(n \cos ^{2} \gamma-p \sin ^{2} \gamma\right) .
\end{aligned}
$$

This form $[\widetilde{A}]$ is a solution of the Einstein-Maxwell equations for arbitary values of the ten parameters $A, h, k, l, m, n, q, p, c, \gamma$, (except when so many of them vanish that the metric becomes singular) subject to the 
conditions that there exists, and the co-ordinate $\mu$ lies in, a range where $\Delta_{\mu}$ is positive, and that the square of the charge parameter, $e^{2}$, as given by (248), is positive or zero. In the case when $e^{2}$ is zero we have a pure vacuum solution determined by nine free parameters including the $\Lambda$ term. In the case where $e^{2}$ is non-zero there is a source free electromagnetic field determined by the vector potential

$$
\begin{aligned}
A= & e \lambda \cos \alpha \frac{\left.\cos \gamma d \chi+\left[\left(c^{2} \mu^{2}+k^{2}+l^{2}\right) \sin \gamma+k\right) c l \mu\right] d \psi}{(c \lambda \cos \gamma+k)^{2}+(c \mu \sin \gamma+l)^{2}} \\
& -e \mu \sin \alpha \frac{\sin \gamma d \chi-\left[\left(c^{2} \lambda^{2}+k^{2}+l^{2}\right) \cos \gamma+2 c k\right] d \psi}{(c \lambda \cos \gamma+k)^{2}+(c \mu \sin \gamma+l)^{2}},
\end{aligned}
$$

where $\alpha$ is an arbitrary complexion angle which does not affect the metric.

Our derivation of the metric $[\widetilde{A}]$ from $[A]$ is of course only valid directly when $c$ is non-zero and $\gamma$ is not an integral multiple of $\pi / 2$, but in consequence of the analyticity, the field equations must continue to be satisfied even when these conditions do not hold. The special cases which arise in this way are just the forms $[\widetilde{B}(+)]$ (when $\sin \gamma=0, c \neq 0$ ), $[\widetilde{B}(-)]$ (when $\cos \gamma=0, c \neq 0$ ), and $[D]$ (when $c=0$ ). These forms are obtained precisely as given in the introduction if, more specially, we set $\gamma=0, k=0, c=1$ to obtain $[\widetilde{B}(+)](l=0$ or $l=1$ giving the subcases $[C(+)]$ and $[B(+)]$ respectively), $\gamma=\pi / 2, l=0, c=1$ to obtain $[\widetilde{B}(-)]$ $(k=0$ or $k=1$ giving the subcases $[C(-)]$ or $[B(-)]$ respectively), and $\gamma=0, k=0, l=1, c=0$ to obtain $[D]$. One can return to the form $[A]$ precisely as given in the introduction by setting $\gamma=\pi / 4, k=0, l=0$, $c=\sqrt{2}$.

The simpler members of the family of solutions obtained here are already well known in other contexts. The vacuum $(e=0)$ members of the class $[D]$ were discovered by KASNER [11], and the complete class has been given by BeRtotri [12] and independently by RoBinson [13]; these spaces are all homogeneous - they have a six parameter isometry group transitive over the whole space. The vacuum members of the classes $[C(\varepsilon)]$ include six cases, corresponding to the possibilities $\varepsilon= \pm 1$, and $h$ positive, zero, or negative, one of these being the Schwarzschild solution from which the other five are obtainable by various simple complex transformations and limiting processes; the six possibilities have been tabulated by EHLERs [10] in the special case where $\Lambda$ vanishes. The charged members of the classes $[C(\varepsilon)]$ include the solution of REISSNER and NorDSTRom together with five other analogously related metrics. In the case where $\Lambda$ vanishes the families $[B(\varepsilon)]$ consist of the space of TAUB (1951) and N.U.T. [15] in the vacuum case and its charged generalisation discovered by BRILL [16] in the case where $\varepsilon$ is +1 and $h$ is positive, together with the five related metrics corresponding to the other possible combinations of $\varepsilon= \pm 1$ and $h$ positive negative or zero; 
the further generalisation to the case where the $\Lambda$ term is non-zero does not appear to have been made before. All the spaces of the classes $[B(\varepsilon)]$ and $[C(\varepsilon)]$ have a four parameter isometry group transitive over the hypersurfaces on which $\lambda^{i}$ is constant. The number of non-redundant parameters in addition to the charge and the $\Lambda$ term is two in the general case $[B(\varepsilon)]$, one in the general case $[C(\varepsilon)]$ (namely the mass in the Schwarzschild solution) and none at all in the case $[D]$.

The largest and most complicated class of the above family is $[A]$. In general the spaces of this class have no isometry beyond the twoparameter group originally postulated. This class consists of two families with three non-redundant parameters in addition to the charge $e$ and the $\Lambda$ term, characterized by the sign of $h$, together with other families with fewer non-redundant parameters in which $h$ vanishes. The most extensive subclass of $[A]$ which has been previously given in explicit form seems to be the family characterized by the conditions $\Lambda=0, h>0$, $h p+q^{2}>0$, which has recently been obtained by Demianski and NEWMAN [17] in a different form by an algebraic trick ${ }^{1}$. When $q=0$ this solution reduces to the charger Kerr solution which was obtained by Newman et al. [18], and which further reduces to the pure vacuum solution of KerR [19] when $e$ vanishes. The Demianski and Newman family includes Brill's charged Taub-NUT space in class $[B(+)]$ as a limiting case. The generalisation to the case where the two inequalities stated above are not satisfied does not seem to have been published explicitly before (although the existence of all these solutions in the pure vacuum case is implicit in the work of KERR [19], and there have been some more detailed studies in unpublished work by KERR, RoBINson, and others). The generalisation to the case where the $\Lambda$ term is present appears to be entirely new.

The work of this paper has been directed in such a way as to obtain maximum algebraic simplicity at all stages, with the result that the global structure even of the previously known spaces is much less obvious in the forms in which they are given here than in the more familiar (but more complicated) forms in which they are usually written. The transformation of these metrics into forms in which the global topology is less obscure (and as a by-product the explicit demonstration of the relationships which have just been described with previously known solutions), together with the investigation of completeness and the construction of analytic extensions of the spaces so obtained will be left for a subsequent paper. The investigation of spaces of the Schrodinger separable canonical forms in which material sources are present will also be postponed.

1 The final metric form published by Demianski and Newman contains several minor algebraic errors. 
Acknowledgements. I should like to thank G. F. R. Ellis, C. W. Mrsner, and D. W. Scrama for several helpful discussion and suggestions.

\section{References}

1. Robertson, H. P.: Math. Ann. 98, 749 (1927).

2. Eisenhart, C. P.: Ann. Math. 35, 284 (1934).

3. CARTER, B.: The global structure of the Kerr family of gravitational fields. Scheduled for publication in: Phys. Rev. Issue No. 5, about October 1968.

4. - Phys. Letters 26 A, 399 (1968).

5. Nambu, Y.: Prog. Theoret. Phys. (Kyoto) 5, 82 (1950).

6. Feynman, R.: Phys. Rev. 80, 940 (1950).

7. Garrod, C.: Rev. Mod. Phys. 38, 489 (1966).

8. Carter, B.: J. Math. Phys. (1968).

9. Papapetrod, A.: Ann. Inst. H. Poincaré 4, 83 (1966).

10. Ehlers, J.: In: Gravitation, ed. L. WitTen. New York: Wiley 1962.

11. KaSner, E.: Trans. Am. Math. Soc. 27, 101 (1925).

12. Bertotri, B.: Phys. Rev. 116, 1331 (1959).

13. Robinson, I.: Bull. Acad. Pol. 7, 351 (1959).

14. TAUB, A.: Ann. Math. 53, 472 (1951).

15. Newman, E., L. Tamborino, and T. Untu: J. Math. Phys. 4, 915 (1963).

16. BrILl, D.: Phys. Rev. 133, B 845 (1963).

17. Demianski, M., and E. Newman: Bull. Acad. Pol. 14, 653 (1966).

18. Newman, E. T., E. Couch, R. Chinnapared, A. Exton, A. Prakash, and R. Torrence: J. Math. Phys. 6, 918 (1965).

19. KERR, R.: Phys. Rev. Letters 11, 237 (1963).

Dr. Brandon Carter

Department of Applied Mathematics and Theoretical Physics

Cambridge, Great Britain 